\title{
Flux Operators of Microdynamical Quantities in a Nonequilibrium Statistical Ensemble Formalism
}

\author{
Justino R. Madureira, Áurea R. Vasconcellos, and Roberto Luzzi* \\ Instituto de Física 'Gleb Wataghin', \\ Universidade Estadual de Campinas, Unicamp \\ 13083-970 Campinas, São Paulo, Brazil \\ Received January 8, 1998
}

\begin{abstract}
It is shown how the closure condition for the set of kinetic equations in Zubarev's Nonequilibrium Statistical Operator Method introduces a series of fluxes of a reference set of densities. These fluxes are the average values, over a Gibbs-like nonequilibrium generalized grandcanonical ensemble, of Hermitian operators for fluxes defined at the microscopic-mechanical level. The equations of evolution for these fluxes (or equivalently for their conjugated Lagrange multipliers) are described.
\end{abstract}

\section{Introduction}

Nowadays nonlinear physics is a frontier area of reserch [1], a situation Werner Heisenberg foresought decades ago [2]. Nonlinearity in the equations of motion which govern the evolution of many-body dynamical systems is well known to be the source of novel and unexpected phenomena which characterize complex behavior in physical systems [3]. This is manifested in the relevant areas of deterministic chaos and selforganization in dissipative systems far from equilibrium [3, 4]. Thermodynamics has a unique role in the question of evolution and eventual selforganization in open systems arbitrarily away from equilibrium. The traditional thermodynamic approach (classical or Onsagerian Thermodynamics) has limitations in dealing with the nonlinear range of irreversible phenomena. To arrive at a satisfactory characterization of nonequilibrium states an enlarged formulation of thermodynamics, incorporating tools and concepts from nonlinear dynamics and stochastic processes, is necessary, leading to what may be termed as thermodynamics of complex systems [5]. Thermodynamics admits several levels of description, as noticed by L. Tisza [6], and one of them, namely, Statistical Thermodynamics is con- sidered as being by itseft richer and the point of departure for a whole array of generalizations. This so-called Gibbs-style Thermodynamics is, of course, based on statistical-mechanical foundations, that is, on the microscopic substrate provided by the many-body dynamics accompanied with theory of probability. This thermodynamics is in a rigorous process of development, its forefront consisting in the so-called Informational Statistical Thermodynamics (IST for short, sometimes referred-to as Information-theoretic Thermodynamics), seemingly initiated by Hobson [7] after the publication of Jaynes' seminal papers on the foundation of statistical mechanics on information theory [8] (see for example $[9,10]$ ). IST is presently based on a statistical nonequilibrium ensemble formalism, or, more precisely, a seemingly powerful, concise and elegant method, established on sound basic principles, consisting in the Nonequilibrium Statistical Operator method (NESOM for short) [11-16]. NESOM may be considered as included within the framework of Jaynes' Predictive Statistical Physics [17], which is based on an approach related to inference from incomplete information. Jaynes' approach has its roots in information theory together with a Bayesian point of view at the probabilistic level [18], and therefore it is referred to as an informational-

\footnotetext{
*Group Home Page:http://www.aurea.ifi.unicamp.br/index.html
} 
theoretic approach. Since the process of construction of the nonequilibrium statistical distribution is based on the principle of maximization of the so-called informational entropy [19, 20], we will refer to the method as the MaxEnt-NESOM [11, 12, 13, 14, 15].

It is our purpose in this communication to describe a particular nonequilibrium statistical ensemble formalism, based on the MaxEnt-NESOM, namely, a generalized nonequilibrium grand-canonical ensemble. As shown in next section it consists of the macroscopic description of the nonequilibrium many-body system in terms of the two typical dynamical quantities, density of particles and density of energy, but in nonequilibrium conditions we need also to introduce the fluxes to all orders of these two densities. The Hermitian operators corresponding to these fluxes are characterized, and finally we briefly describe the use of this ensemble for the construction of a nonclassical thermo-hydrodynamics.

\section{Nonequilibrium Grand- Canonical Ensemble}

The first, and fundamental, step in MaxEnt-NESOM is the choice of the basic set of variables deemed appropriate for the characterization of the macroscopic state of the system. This involves a description in terms of, say, the mechanical quantities $\left\{\hat{P}_{j}(\mathbf{r})\right\}, j=1,2, \ldots$, with the upper circumflex indicating Hermitian operators, and the dependence on the space coordinate $\mathbf{r}$ indicates the local density of the corresponding dynamical quantity $\hat{P}_{j}$. The MaxEnt-NESOM nonequilibrium statistical operator will be denoted by $\rho_{\epsilon}(t)$, and Zubarev's approach is consistently used. The thermodynamics (macroscopic or mesoscopic) state is characterized by a point in Gibbs - or thermodynamic state - space given, at time $t$, by the set of macrovariables $\left\{Q_{j}(\mathbf{r}, t)\right\}, j=1,2, \ldots$, which are the averages of the $\hat{P}_{j}(\mathbf{r})$, i.e., $Q_{j}(\mathbf{r}, t)=\operatorname{Tr}\left\{\hat{P}_{j}(\mathbf{r}) \rho_{\epsilon}(t)\right\}$. The choice of the basic variables is assisted by the fundamental Bogoliubov procedure of contraction of description based on a hierarchy of relaxation times [21], and - to it related - the ideas put forward by, among others, Mori [22], Zubarev [11], and Peletminskii [16], introducing a separation of the total Hamiltonian into two parts, namely,

$$
\hat{H}=\hat{H}_{o}+\hat{H}^{\prime}
$$

where $\hat{H}_{0}$ is the "relevant" (or secular) part composed of the Hamiltonian for the free subsystems and a part of the interactions, namely, those strong enough to have associated relaxation processes with very short relaxation times (meaning those much smaller than the characteristic time scale of the experiment (or resolution time). The other term, $\hat{H}^{\prime}$, contains the interactions related to long-time relaxation mechanisms. The symmetry characteristics of the strong interactions depend on the problem under consideration: The required symmetry - to be called Zubarev-Peletminskii symmetry condition - is that

$$
\frac{1}{i \hbar}\left[\hat{P}_{j}, \hat{H}_{o}\right]=\sum_{k} \alpha_{j k} \hat{P}_{k}
$$

where the left side is the commutator of $\hat{P}_{j}$ and $\hat{H}_{o}$, and $\alpha_{j k}$ are, in an appropriate quantum representation, real numbers. It should be noticed that quantities $\hat{P}_{j}$ can be dependent on the space variable, i.e., when they are densities and then quantities $\alpha$ can also be differential operators.

Equation (2) provides a closure-like condition for the choice of the set of variables. In reference [23] this procedure is related to the question of the choice of the basic variables in phenomenological irreversible thermodynamics. Practical use of the formalism usually requires to introduce an appropriated truncation procedure (see second of references [23]) along the chain that application of the method produces, or an asymptotic expansion yielding renormalized coefficients. It ought to be noticed that Eq.(2) also encompasses the case of quantities $\hat{P}$ such that they have associated null coefficients $\alpha$; i.e., they are constants of motion under the dynamics generated by $\hat{H}_{o}$. Accordingly they are acceptable basic variables, and $\hat{H}_{0}$ itself falls under this condition, and should be always present in the basic set.

Assuming that the basic set $\left\{\hat{P}_{j}(\mathbf{r})\right\}$ has been chosen, the nonequilibrium statistical operator is built in MaxEnt-NESOM, using the principle of maximization of the statistical-informational entropy, with fading memory and an ad hoc hypothesis which introduce from the outset irreversible evolution from an initial condition of preparation of the system: for details see references $[12,13,15,24]$. Summarizing for later use, in the 
particular case of Zubarev's approach to the MaxEntNESOM $[11,12]$ (by far the most concise and practical one, and founded on sound physical basis) the nonequilibrium statistical operator is given by

$$
\rho_{\epsilon}(t)=\exp \left\{\ln \bar{\rho}(t, 0)-\int_{-\infty}^{t} d t^{\prime} e^{\epsilon\left(t^{\prime}-t\right)} \frac{d}{d t^{\prime}} \ln \bar{\rho}\left(t^{\prime}, t^{\prime}-t\right)\right\}
$$

where $\bar{\rho}$ is an auxiliary operator (or coarse-grained part of $\rho_{\epsilon}(t)$ ) - of large practical relevance in the theory - given by the Gibbsian-like generalized nonequilibrium canonical distribution

$$
\bar{\rho}(t, 0)=\exp \left\{-\phi(t)-\sum_{j} \int d^{3} r F_{j}(\mathbf{r}, t) \hat{P}_{j}(\mathbf{r})\right\},
$$

and

$$
\bar{\rho}\left(t^{\prime}, t^{\prime}-t\right)=\exp \left\{-\frac{1}{i \hbar}\left(t^{\prime}-t\right) \hat{H}\right\} \bar{\rho}\left(t^{\prime}, 0\right) \exp \left\{\frac{1}{i \hbar}\left(t^{\prime}-t\right) \hat{H}\right\}
$$

In Eq.(4), $\phi(t)$ ensures the normalization of $\bar{\rho}$, and the $F_{j}(t)$ (as well as $\phi$ ) are the Lagrange multipliers that MaxEnt introduces. In Eq.(3) $\epsilon$ is a positive infinitesimal that goes to zero after the calculation of the averages is performed; this implies to introduce Bogoliubov's quasi-averages procedure [25], a symmetrybreaking process, in this case corresponding to a breaking of the time-reversal symmetry in Liouville equation [12, 13, 14, 24]. Space dependence has been explicitly introduced, and in Eq.(3) it is clear the presence of retro-effects but with fading memory, where quantities $\hat{P}_{j}$ are given in the time-dependent Heisenberg representation. We recall that $\rho_{\epsilon}(t)$ does satisfy Liouville equation, but the presence of the kernel $\exp \{\epsilon(t-t)\}$ leads in the calculation of averages and transport coefficients to a time-smoothing procedure. Variables $F_{j}(t)$ are related to the macrovariables $Q_{j}$ through the relations

$$
Q_{j}(\mathbf{r}, t)=\operatorname{Tr}\left\{\hat{P}_{j}(\mathbf{r}) \rho_{\epsilon}(t)\right\}
$$

with $j=1,2, \ldots$, and the method allows for the construction of a quantum nonlinear, nonlocal local in space, and memory dependent kinetic theory describing information on the dissipative evolution of the macroscopic nonequilibrium state of the system $[11,12,13,26]$.

Let us consider the construction of the MaxEntNESOM nonlinear quantum kinetic theory. First, it should be noticed that the equations of evolution for the basic variables are given by

$$
\frac{\partial}{\partial t} Q_{j}(\mathbf{r}, t)=\operatorname{Tr}\left\{\frac{1}{i \hbar}\left[\hat{P}_{j}(\mathbf{r}), \hat{H}\right] \rho_{\epsilon}(t)\right\},
$$

that is to say, they are the average over the nonequilibrium ensemble of the corresponding Heisenberg equation of motion for quantities $\hat{P}_{j}(\mathbf{r})$. Equations (6), taking into account that $j=1,2, \ldots$, constitute, in general, a coupled set of integro-differential equations of formidable proportions. But it is possible to obtain an alternative expression for the right hand side of Eq.(6) easier to handle mathematically and allowing to obtain a more clear physical picture of the dissipative processes that develop in the media [12-14,26]. A price is paid, consisting in the fact that we obtain an infinite series of collision operators associated to two, three, etc, collisional processes, but at the same time permits evaluation of the different contributions and to introduce a cut-off in the series expansion. This, of course, amounts to an approximation and therefore each particular case requires an evaluation of its validity [27]. 
We note that the equations of evolution for the macrovariables which characterize the macroscopic nonequilibrium state of the system, Eq.(6), can be rewritten in the form

$$
\frac{d}{d t} Q_{j}(t)=J_{j}^{(0)}(t)+J_{j}^{(1)}(t)+\mathcal{J}_{j}(t)
$$

where

$$
\begin{gathered}
\left.J_{j}^{(0)}(t)=\operatorname{Tr}\left\{\frac{1}{i \hbar}\left[\hat{P}_{j}, H_{0}\right] \bar{\rho}(t, 0)\right]\right\}, \\
\left.J_{j}^{(1)}(t)=\operatorname{Tr}\left\{\frac{1}{i \hbar}\left[\hat{P}_{j}, H^{\prime}\right] \bar{\rho}(t, 0)\right]\right\}, \\
\left.\mathcal{J}_{j}(t)=\operatorname{Tr}\left\{\frac{1}{i \hbar}\left[\hat{P}_{j}, H^{\prime}\right] \rho_{\epsilon}^{\prime}(t)\right]\right\},
\end{gathered}
$$

after writing $\rho_{\epsilon}(t)=\bar{\rho}(t, 0)+\rho_{\epsilon}^{\prime}(t)$ and using Eqs.(1) and (2) [11-16,26,28,29]. Equation (7) is a far-reaching generalization of Mori's equations (see [13] and [28]). The first two terms, $J_{j}^{(0)}$ and $J_{j}^{(1)}$ are, in Mori's terminology, precession terms, and the collision operator of Eq.(8c) is, differently to the original Mori's theory, a highly nonlinear term in the basic variables; also it is nonlocal in space (for simplicity we have omitted the possible space dependence of quantities $\hat{P}_{j}$ ), and it contains memory effects (of a fading character).

The collision operator of Eq.(8c) is extremely difficult to handle in practical calculations. In reference [26] we show that, using the properties defined by Eqs.(1) and (2), the complicated collision operator of Eq.(8c) can be rewritten in terms of an infinite series of collision integrals which are instantaneous in time (given as averages over the auxiliary NESO of Eq. (4a) at the time of measurement) and organized in increasing powers $n$ of the interaction strengths, namely,

$$
\frac{d}{d t} Q_{j}(t)=J_{j}^{(0)}(t)+J_{j}^{(1)}(t)+\sum_{n=2}^{\infty} J_{j}^{(n)}(t)
$$

with the construction of quantities $J_{j}^{(n)}$ described in reference [26].

The form of the collision operator given by Eq.(9) permits to introduce approximations by means of a truncation of the series of partial collision operators in a given order of the interaction. The lowest order that introduces relaxation effects is a truncation in second order in the interaction strengths: it consists in retaining only binary collisions in a Markovian (memoryless) approximation [11, 16, 29].
Let us specialize the theory just described for the construction of a generalized nonequilibrium grandcanonical ensemble. For that purpose consider a system of many-bosons or fermions, described in an individual particle representation (the typical situation in solid state matter), and let $\varepsilon_{\mathbf{k}_{\sigma}}$ be the corresponding single-particle energies ( $\mathbf{k}$ is the crystalline momentum runing over the Brillouin zone and $\sigma$ is the spin index). In equilibrium conditions the grand-canonical distribution depends on the energy and particle-number operators. In nonequilibrium conditions, analogously, we begin introducing as first basic dynamical variables the density of energy $\hat{h}(\mathbf{r})$ and of the particle-number $\hat{n}(\mathbf{r})$. However, for practical purposes it is more convenient to work with their Fourier transforms of wavevector $\mathbf{Q}$, that is, to perform the calculations in reciprocal space. Then we have that

$$
\begin{gathered}
\hat{n}(\mathbf{Q})=\sum_{\mathbf{k}} \hat{n}_{\mathbf{k} \mathbf{Q}}, \\
\hat{h}(\mathbf{Q})=\sum_{\mathbf{k}} E_{\mathbf{k} \mathbf{Q}}{ }^{\hat{n}} \mathbf{k} \mathbf{Q},
\end{gathered}
$$

where

$$
E_{\mathbf{k Q}}=\frac{1}{2}\left(\varepsilon_{\mathbf{k}+\frac{1}{2} \mathbf{Q}}+\varepsilon_{\mathbf{k}-\frac{1}{2} \mathbf{Q}}\right)
$$

and

$$
{ }^{\hat{n}_{\mathbf{k}} \mathbf{Q}}={ }^{\dagger}{ }_{\mathbf{k}+\frac{1}{2}}^{\dagger} \mathbf{Q}{ }^{c} \mathbf{k}-\frac{1}{2} \mathbf{Q}
$$

is Wigner-Landau single-particle dynamical operator. Moreover, for simplicity - but without losing generality - we have taken plane wave states, the volume of the system is taken equal to 1 , and we disregard the spin index. As usual $c^{\dagger}(c)$ are creation (annihilation) operators in states $|\mathbf{k}\rangle$, for either fermion or boson system.

We write for the system Hamiltonian, taken in the form given by Eq.(1),

$$
\begin{aligned}
& \hat{H}_{o}=\sum_{\mathbf{k}} \varepsilon_{\mathbf{k}} c_{\mathbf{k}}^{\dagger} c_{\mathbf{k}}, \\
& \hat{H}^{\prime}=\hat{H}_{S}^{\prime}+\hat{H}_{B}^{\prime},
\end{aligned}
$$

where $\hat{H}_{S}^{\prime}$ and $\hat{H}_{B}^{\prime}$ accounts for the interactions of the single-particle with external sources and thermal baths 
respectively (details are not necessary for our purposes here).

Next, we need to look for the verification of the closure condition (Zubarev-Peletminskii symmetry condition) of Eq.(2): This introduces, as anticipated in the Introduction, the fluxes of all order of the two basic densities in the picture. First we calculate

$$
\begin{gathered}
\frac{1}{i \hbar}\left[\hat{n}(\mathbf{Q}), \hat{H}_{o}\right]=i \sum_{\mathbf{k}} \Delta \omega_{\mathbf{k} \mathbf{Q}} \hat{n}_{\mathbf{k} \mathbf{Q}}, \\
\frac{1}{i \hbar}\left[\hat{h}(\mathbf{Q}), \hat{H}_{o}\right]=i \sum_{\mathbf{k}} E_{\mathbf{k} \mathbf{Q}} \Delta \omega_{\mathbf{k} \mathbf{Q}}{ }^{\hat{n}} \mathbf{k} \mathbf{Q},
\end{gathered}
$$

where

$$
\Delta \omega_{\mathbf{k} \mathbf{Q}}=\omega_{\mathbf{k}+\frac{1}{2}} \mathbf{Q}-\omega_{\mathbf{k}-\frac{1}{2} \mathbf{Q}} \quad ; \quad \hbar \omega_{\mathbf{k}}=\varepsilon_{\mathbf{k}} .
$$

As shown elsewhere [30] (and the case which includes interparticle interaction is dealt with in [31]), we can write

$$
\Delta \omega_{\mathbf{k Q}}=\mathrm{Q} \cdot \mathbf{u}(\mathbf{k Q})
$$

where quantity $\mathbf{u}$, with units of velocity, is given by

$$
\mathbf{u}(\mathbf{k Q})=\sum_{l=0}^{\infty} \frac{1}{(2 l+1) !}\left(\frac{\mathbf{Q}}{2} \cdot \nabla_{\mathbf{k}}\right)^{2 l} \nabla_{\mathbf{k}} \omega_{\mathbf{k}},
$$

which has a very peculiar form, namely, the first contribution $(l=0)$ is the group velocity of the singleparticle in state $|\mathbf{k}\rangle$, followed by the curvature of this k-dependent group velocity, etc. Hence, Eqs.(15) take the form

$$
\begin{aligned}
& \frac{1}{i \hbar}\left[\hat{n}(\mathbf{Q}), H_{o}\right]=i \mathbf{Q} \cdot \hat{\mathbf{I}}_{n}(\mathbf{Q}) \\
& \frac{1}{i \hbar}\left[\hat{h}(\mathbf{Q}), H_{0}\right]=i \mathbf{Q} \cdot \hat{\mathbf{I}}_{h}(\mathbf{Q})
\end{aligned}
$$

where

$$
\begin{gathered}
\hat{\mathbf{I}}_{n}(\mathbf{Q})=\sum_{\mathbf{k}} \mathbf{u}(\mathbf{k Q}) \hat{n}_{\mathbf{k Q}} \\
\hat{\mathbf{I}}_{h}(\mathbf{Q})=\sum_{\mathbf{k}} E_{\mathbf{k Q}} \mathbf{u}(\mathbf{k Q}) \hat{n}_{\mathbf{k} \mathbf{Q}} .
\end{gathered}
$$

According to the closure condition both are incorporated as basic variables. Next, the rule of Eq.(2) is applied to them, to obtain

$$
\frac{1}{i \hbar}\left[\hat{\mathbf{I}}_{n}(\mathbf{Q}), H_{\circ}\right]=i \mathbf{Q} \cdot \hat{I}_{n}^{[2]}(\mathbf{Q})
$$

$$
\frac{1}{i \hbar}\left[\hat{\mathbf{I}}_{h}(\mathbf{Q}), H_{0}\right]=i \mathbf{Q} \cdot \hat{I}_{h}^{[2]}(\mathbf{Q})
$$

where

$$
\begin{gathered}
\hat{I}_{n}^{[2]}(\mathbf{Q})=\sum_{\mathbf{k}}[\mathbf{u}(\mathbf{k Q}) \mathbf{u}(\mathbf{k Q})] \hat{n}_{\mathbf{k} \mathbf{Q}}, \\
\hat{I}_{h}^{[2]}(\mathbf{Q})=\sum_{\mathbf{k}} E_{\mathbf{k} \mathbf{Q}}[\mathbf{u}(\mathbf{k Q}) \mathbf{u}(\mathbf{k Q})] \hat{n}_{\mathbf{k} \mathbf{Q}},
\end{gathered}
$$

are second rank tensors (the term [uu] between square brackets stands for tensorial product of vectors) which constitute the second order fluxes (or flux of the flux) of density and energy density operators, respectively. Therefore, they are to be included in the basic set, and so on, that is, the closure condition commands that the fluxes of all order of the two basic densities must be considered as basic dynamical variables. The basic set is then composed by

$$
\left\{\hat{h}(\mathbf{Q}), \hat{n}(\mathbf{Q}), \hat{\mathbf{I}}_{h}(\mathbf{Q}), \hat{\mathbf{I}}_{n}(\mathbf{Q}), \hat{I}_{h}^{[r]}(\mathbf{Q}), \hat{I}_{n}^{[r]}(\mathbf{Q})\right\}
$$

where $r \geq 2$ indicates the order (and also tensorial rank, with $r=1$ being the vectorial ones) of the fluxes, given by

$$
\hat{I}_{n}^{[r]}(\mathbf{Q})=\sum_{\mathbf{k}} u^{[r]}(\mathbf{k Q}) \hat{n}_{\mathbf{k}} \mathbf{Q}
$$

$$
\hat{I}_{h}^{[r]}(\mathbf{Q})=\sum_{\mathbf{k}} E_{\mathbf{k Q}}{ }^{u^{[r]}(\mathbf{k Q}) \hat{n}_{\mathbf{k}} \mathbf{Q}}
$$

where

$$
u^{[r]}(\mathbf{k Q})=[\mathbf{u}(\mathbf{k Q}) \ldots(r \text { times }) \ldots \mathbf{u}(\mathbf{k Q})]
$$

indicating tensorial product of $r$ vectors $\mathbf{u}$, producing a tensor of rank $r$ [30].

Therefore, in terms of the basic set of dynamical variables of Eq.(23), the auxiliary (coarse-grained or "frozen" quasi-equilibrium) statistical operator of Eq. (4a) is in this case given by 


$$
\begin{aligned}
\bar{\rho}(t, 0)=\exp \{ & -\phi(t)-\sum_{\mathbf{Q}}\left[F_{h}(\mathbf{Q}, t) \hat{h}(\mathbf{Q})+F_{n}(\mathbf{Q}, t) \hat{n}(\mathbf{Q})\right] \\
& -\sum_{\mathbf{Q}}\left[\boldsymbol{\alpha}_{h}(\mathbf{Q}, t) \cdot \hat{\mathbf{I}}_{h}(\mathbf{Q})+\boldsymbol{\alpha}_{n}(\mathbf{Q}, t) \cdot \hat{\mathbf{I}}_{n}(\mathbf{Q})\right] \\
& \left.-\sum_{\mathbf{Q}} \sum_{r \geq 2}\left[\alpha_{h}^{[r]}(\mathbf{Q}, t) \times \hat{I}_{h}^{[r]}(\mathbf{Q})+\alpha_{n}^{[r]}(\mathbf{Q}, t) \times \hat{I}_{n}^{[r]}(\mathbf{Q})\right]\right\},
\end{aligned}
$$

where $x$ stands for fully contracted product of tensors, and we have introduced the corresponding set of associated Lagrange multipliers indicated by

$$
\left\{F_{h}(\mathbf{Q}, t), F_{n}(\mathbf{Q}, t), \boldsymbol{\alpha}_{h}(\mathbf{Q}, t), \boldsymbol{\alpha}_{n}(\mathbf{Q}, t), \alpha_{h}^{[r]}(\mathbf{Q}, t), \alpha_{n}^{[r]}(\mathbf{Q}, t)\right\} .
$$

First, as already noticed, these Lagrange multipliers constitute a set of intensive variables, that, alternatively, completely describe the nonequilibrium thermodynamic state of the system. They are related to the basic set of macrovariables by the relations (which are the equivalent of equations of state in arbitrary nonequilibrium conditions)

$$
\begin{aligned}
& n(\mathbf{Q}, t)=\operatorname{Tr}\{\hat{n}(\mathbf{Q}) \bar{\rho}(t, 0)\}=-\frac{\delta \phi(t)}{\delta F_{n}(\mathbf{Q}, t)}, \\
& h(\mathbf{Q}, t)=\operatorname{Tr}\{\hat{h}(\mathbf{Q}) \bar{\rho}(t, 0)\}=-\frac{\delta \phi(t)}{\delta F_{h}(\mathbf{Q}, t)},
\end{aligned}
$$

etc., where we have used that $\rho_{\epsilon}$ and $\bar{\rho}$, at each time $t$, define the same average values [11-15] and $\delta$ stands for functional differential [33]. As already noticed, the basic set of macrovariables which define the nonequilibrium thermodynamic space of states in IST (in this case we can refer to it as the Gibbs' grand-canonical space of macrostates) is given by the average values over the nonequilibrium ensemble of the microdynamical variables of Eq.(23), i.e.,

$$
\begin{aligned}
& h(\mathbf{Q}, t)=\operatorname{Tr}\left\{\hat{h}(\mathbf{Q}) \rho_{\epsilon}(t)\right\}, \\
& n(\mathbf{Q}, t)=\operatorname{Tr}\left\{\hat{n}(\mathbf{Q}) \rho_{\epsilon}(t)\right\}, \\
& I_{h(n)}^{[r]}(\mathbf{Q}, t)=\operatorname{Tr}\left\{\hat{I}_{h(n)}^{[r]}(\mathbf{Q}) \rho_{\epsilon}(t)\right\},
\end{aligned}
$$

that is, they compose the set

$$
\left\{h(\mathbf{Q}, t), n(\mathbf{Q}, t), \mathbf{I}_{h}(\mathbf{Q}, t), \mathbf{I}_{n}(\mathbf{Q}, t), I_{h}^{[r]}(\mathbf{Q}, t), I_{n}^{[r]}(\mathbf{Q}, t)\right\},
$$

Hence, we have constructed a nonequilibrium grandcanonical nonequilibrium statistical operator, which, we stress is the $\rho_{\epsilon}(t)$ of Eq.(3) built using the auxiliary operator of Eq.(26). In Ref. [30] it is shown that this statistical operator goes over the well known grand- canonical distribution in the limit when equilibrium is achieved. 


\section{Nonclassical Hydrodynam- ics}

This nonequilibrium grand-canonical operator describes a fluid of particles and provides foundation for a nonclassical hydrodynamics. In fact, the equations of evolution, Eqs.(9), for the basic variables of Eq.(29), when we transform back to the direct space, are given

$$
\begin{gathered}
\frac{\partial}{\partial t} h(\mathbf{r}, t)+\nabla \cdot \mathbf{I}_{h}(\mathbf{r}, t)=\mathcal{J}_{h}(\mathbf{r}, t), \\
\frac{\partial}{\partial t} n(\mathbf{r}, t)+\nabla \cdot \mathbf{I}_{n}(\mathbf{r}, t)=\mathcal{J}_{n}(\mathbf{r}, t), \\
\frac{\partial}{\partial t} \mathbf{I}_{h}(\mathbf{r}, t)+\nabla \cdot I_{h}^{[2]}(\mathbf{r}, t)=\mathcal{J}_{h}(\mathbf{r}, t), \\
\frac{\partial}{\partial t} \mathbf{I}_{n}(\mathbf{r}, t)+\nabla \cdot I_{n}^{[2]}(\mathbf{r}, t)=\mathcal{J}_{n}(\mathbf{r}, t), \\
\frac{\partial}{\partial t} I_{h}^{[r]}(\mathbf{r}, t)+\nabla \cdot I_{h}^{[r+1]}(\mathbf{r}, t)=\mathcal{J}_{h}^{[r]}(\mathbf{r}, t), \\
\frac{\partial}{\partial t} I_{n}^{[r]}(\mathbf{r}, t)+\nabla \cdot I_{n}^{[r+1]}(\mathbf{r}, t)=\mathcal{J}_{n}^{[r]}(\mathbf{r}, t),
\end{gathered}
$$

with $r \geq 2$, and, clearly, if the collision operators

$$
\mathcal{J}_{h}(\mathbf{r}, t)=\operatorname{Tr}\left\{\frac{1}{i \hbar}\left[\hat{h}(\mathbf{r}), H^{\prime}\right] \rho_{\epsilon}^{\prime}(t)\right\}
$$

etc., are neglected we obtain the conservation equation for each quantity, namely for the densities and their fluxes of all order.

Equations (30) provide the thermo-hydrodynamics of the nonequilibrium many-body system, which, clearly, constitute an infinite set of coupled equations. Evidently, a practical use requires to introduce a truncation procedure, meaning the analog of the one in the Hilbert-Chapmann-Enskog approach to Boltzmann equation. This is discussed in a forthcoming article [27], and a particular case - a photoinjected plasma in semiconductors - is considered in [34].

Moreover, as a general rule the extremely complicated collision operator $\mathcal{J}$ in Eqs.(30), can be calculated in the Markovian approximation to the MaxEntNESOM kinetic theory, consisting in that in Eq.(9) one retains only the first contribution $J^{(2)}$, which accounts for instantaneous two-particle collision-like processes, ignoring the influence of the past irreversible evolution of the macrostate of the system. Without going into further details, we noticed that in the above said Markovian approximation and in the limit of very weak fluxes and densities varying smoothly in space and time (examples related to the photoinjected plasma in semiconductors are given in $[35,36,37]$ ), a truncation procedure in which we retain only the densities of energy and of particles and their first (vectorial)fluxes, their equations of evolution are to a good approximation given by

$$
\begin{gathered}
\frac{\partial}{\partial t} h(\mathbf{r}, t)+\nabla \cdot \mathbf{I}_{h}(\mathbf{r}, t)=-\theta_{h}^{-1} h(\mathbf{r}, t), \\
\frac{\partial}{\partial t} \mathbf{I}_{h}(\mathbf{r}, t)+L_{h} \nabla h(\mathbf{r}, t)=-\theta_{h}^{-1} \mathbf{I} \mathbf{I}_{h}(\mathbf{r}, t),
\end{gathered}
$$

where the coefficients $\theta_{h}$ and $\theta_{h} \mathbf{I}$ play the role of relaxation times and $L_{h}$ is a kinetic coefficient, and similarily we obtain the evolution for the density of particles, changing $h$ by $n$ in Eqs.(32a) and (32b). We have neglected cross-kinetic terms coupling the motion of mass and of energy, meaning that thermo-striction effects are disregarded.

Differentiating in time Eq.(32a), and using Eqs.(32b), we find an equation of evolution for the density of energy in the form

$$
\left[\frac{1}{c_{h}^{2}} \frac{\partial^{2}}{\partial t^{2}}+\frac{1}{D_{h}} \frac{\partial}{\partial t}-\nabla^{2}\right] h(\mathbf{r}, t)=-\frac{h(\mathbf{r}, t)}{\Lambda^{2}},
$$

which is of the type of the hyperbolic-type telegraphist equation of classical electrodynamics, but with an additional term on the right. In this Eq. $(33), c_{h}=L_{h}^{1 / 2}$ is the velocity of propagation in the motion of energy, $\Lambda^{2}=c_{h}^{2} \theta_{h} \theta_{h} \mathbf{I}$ (with dimensions of $\mathrm{cm}^{2}$ ), and $D_{h}=c_{h}^{2} \theta_{*}$, with $\theta_{*}^{-1}=\theta_{h}^{-1}+\theta_{h \mathbf{I}}^{-1}$, is a diffusion coefficient. This equations, once we disregard the right hand side, is also of the form of the one that is derived within the framework of phenomenological Extended Irreversible Thermodynamics [38].

Equation (33) implies in second sound propagation in the carrier system. As known [39], such motion is composed of a superposition of diffusive motion (involving the long wavelengths) and damped undulated motion (at intermediate to short wavelengths); these characteristics are, for example, evidenced in experiments related to the techno-industrial process of thermal stereolithography [40].

A MaxEnt-NESOM nonclassical hydrodynamics and a generalized nonclassical Navier-Stokes equation, 
for fluids described at the classical level of mechanics, are presented in [41] and [42] respectively.

\section{Concluding Remarks}

We have described how the nonequilibrium ensemble formalism MaxEnt-NESOM - which can be placed under the umbrella of Jaynes's Preditive Statistical Mechanics - provides a quite convenient framework for dealing with the Statistical Thermodynamics of manybody systems away from equilibrium. In this communication we have paid particular attention to the construction of a generalized nonequilibrium grandcanonical statistical operator. As shown according to the tenets of the formalism once the local density of particle and of energy are introduced, the closure condition requires the introduction of their fluxes to all orders. These fluxes are given at the mechanical level in terms of the single-particle dynamical operator (its average over the statistical ensemble provides nonequilibrium Wigner-Landau single-particle matrices), which are built in terms of a generating vector [cf. Eq.(18)], with dimensions of velocity, and completely determined by the quantum-mechanical energy dispersion relation of the single-particles. It involves a series expansion in terms of increasing powers in the reciprocal of the wavelength, the first contribution being the single-particle wave-packet group velocity (for a simply quadratic dispersion relation is $\hbar \mathbf{k} / m$, where $\hbar \mathbf{k}$ is the eigenvalue of the single-particle linear momentum and $m$ the mass). The average over the nonequilibrium ensemble of these mechanical fluxes are the macroscopic fluxes of the NESOM-based thermo-hydrodynamics [cf. Eq.(29)].

The variational method that - similarly to the case in equilibrium - provides the nonequilibrium statistical operator, introduces the corresponding Lagrange multipliers associated to these fluxes [cf. Eq.(27)]. We have already stressed that these Lagrange multipliers define a set of intensive nonequilibrium thermodynamic variables which provide a complete description of the nonequilibrium thermodynamic state of the system, as do the complete set of specific basic variables of Eq.(29). They are completely defined in terms of the specific basic variables, and, since the latter have associated a ki- netic theory of large scope, we can obtain a complete set of equations of evolution for the Lagrange multipliers. As a matter of fact, this is usually the most convenient approach, because these Lagrange multipliers can be accessible to measurement in experiments (see for example [43] and [44]). A partial physical interpretation of these Lagrange multipliers is possible: First, by close analogy with equilibrium and local equilibrium thermodynamics, the Lagrange multipliers associated to the density of energy and density of particles, are, respectively, considered as a reciprocal of a nonequilibrium temperature-like field dubbed a quasi-temperature [43], and a nonequilibrium chemical potential-like variable, dubbed a quasi-chemical potential, divided by the quasi-temperature [44]. Those Lagrange multipliers associated to the fluxes, can be interpreted as a drift velocity fields divided by the quasitemperature, with the one associated to the first flux of particles (proportional to the linear momentum field) being the usual kinetic drift velocity field [28, 30]. We recall that in Ref. [30] we have shown how the nonequilibrium statistical operator becomes the usual Gibbs' grand-partition distribution once the system attains final full equilibrium.

As noticed, this nonequilibrium grand-canonical statistical operator provides a quite convenient foundation for the construction of a nonclassical thermohydrodynamics of large scope. A short illustration has been provided however in strongly simplified conditions.

A brief report of these results was orally presented as a communication in the $\mathrm{V}$ Latin American Workshop on Nonlinear Phenomena (Canela, RS, Brazil, October 1997) [45].

\section{Acknowledgments}

We acknowledge finantial support to our Group that in different opportunities was provided by the State of São Paulo Research Foundation (FAPESP); the National Research Council (CNPq); Unicamp Foundation (FAEP); the Ministry of Planning (FINEP); IBMBrasil; the John Simon Guggenheim Memorial Foundation (New York, USA). One of the authors (JRM) 
is a FAPESP Post-Doctoral Fellow, and the other two (ARV and RL) are CNPq Research Fellows.

\section{References}

[1] P. Davies, Introductory chapter in The New Physics, edited by P. Davies (Cambridge Univ. Press, Cambridge, 1989).

[2] W. Heisenberg, Nonlinear Problems in Physics, Phys. Today, May 1967, pp. 27-33.

[3] J. A. Krumhansl, Nonlinear Science: Towards the Next Frontiers, Physica D 68, 97 (1993).

[4] G. Nicolis, Physics of Far-from-equilibrium Systems and Selforganization, in The New Physics, edited by P. Davies (Cambridge Univ. Press, Cambridge, 1989).

[5] G. Nicolis, Introductory Remarks: Thermodynamics Today, Physica A 213, 1 (1995).

[6] L. Tisza, Concluding Remarks in Thermodynamics: Hystory and Philosophy, edited by K. Martinas, L. Ropolyi, and P. Szegedi (World Scientific, Singapore, 1991).

[7] A. Hobson, Irreversibility and Information in Mechanical Systems, J. Chem. Phys. 45, 1352 (1966).

[8] E. T. Jaynes, Information Theory and Statistical Mechanics I, Phys. Rev. 106, 620 (1957); II: ibid. 108171 (1957).

[9] L. S. Garcia-Colin, A R. Vasconcellos, and R. Luzzi, On Informational Statistical Thermodynamics, J. NonEquilib. Thermodyn. 19, 24 (1994).

[10] R. Luzzi, A. R. Vasconcellos, and J. G. Ramos, Statistical Foundations of Irreversible Thermodynamics, IFGW-Unicamp Internal Report (1997), and Fortschr. Phys. / Prog. Phys., in press (1998).

[11] D. N. Zubarev, Nonequilibrium Statistical Thermodynamics (Consultants Bureau, New York, 1974).

[12] D. N. Zubarev, V. N. Morosov, and G. Röpke, Statistical Mechanics of Nonequilibrium Phenomena, Vol.1: Basic Concepts, Kinetic Theory (Akademie Verlag, Berlin, 1996).

[13] R. Luzzi and A. R. Vasconcellos, On the Nonequilibrium Statistical Operator Method, Fortschr. Phys. / Prog. Phys., 38, 887 (1990).

[14] J. G. Ramos, A. R. Vasconcellos, and R. Luzzi, A Classical Approach in Predictive Statistical Mechanics: A Generalized Boltzmann Formalism, Fortschr. Phys. / Prog. Phys. 43, 265 (1995).

[15] R. Luzzi, A. R. Vasconcellos, and J. G. Ramos, Considerations on a Nonequilibrium Ensemble Formalism, IFGW-Unicamp Internal Report (1997), and future publication.

[16] A. I. Akhiezer and S. V. Peletminskii, Methods of Statistical Physics (Pergamon, Oxford, 1981).

[17] E. T. Jaynes, Predictive Statistical Mechanics, in Frontiers of Nonequilibrium Statistical Physics, edited by G. T. Moore and M. O. Scully (Plenum, New York, 1986)
[18] P. W. Anderson, The Reverend Thomas Bayes, Needles in a Haystack, and the Fifth Force, Phys. Today 45 (1), 9 (1992).

[19] E. T. Jaynes, reprinted articles and notes in E. T. Jaynes Papers on Probability, Statistics, and Statistical Physics, edited by D. Rosenkrantz (Reidel, Dordrecht, 1983).

[20] J. N. Kapur and H. K. Kesavan, Entropy Optimization Principles with Applications (Academic, San Diego, 1992).

[21] N. N. Bogoliubov, Problems of a Dynamical Theory in Statistical Physics, in Studies in Statistical Mechanies I, edited by J. de Boer and G. E. Uhlenbeck (North Holland, Amsterdam, 1962); for additional discussions and applications see: G. E. Uhlenbeck, in Lectures in Statistical Mechanics, edited by M. Kac (Am. Math. Soc., Providence, RI, 1963); L. L. Buishvili and M. D. Sviadadze, On the Quasithermodynamic Theory of Magnetic Relaxation, Physica 59, 697 (1972); A. R. Vasconcellos, A. C. Algarte, and R. Luzzi, On the Relaxation Time Hierarchy in Dissipative Systems: An Example from Semiconductor Physics, Physica A166, 517 (1990).

[22] H. Mori, Transport, Collective Motion, and Brownian Motion, Prog. Theor. Phys. (Japan) 33, 423 (1965).

[23] A. R. Vasconcellos, R. Luzzi, and L. S. Garcia-Colin, A Microscopic Approach to Irreversible Thermodynamics I, Phys. Rev. E 43, 6022 (1991); II. ibid E 43, 6633 (1991); R. Luzzi and A. R. Vasconcellos, The Basic Principles of Irreversible Thermodynamics in an Informational Statistical Approach, Physica A, in press (1997).

[24] J. T. Alvarez-Romero and L. S. Garcia-Colin, The Foundation of Informational Statistical Thermodynamics Revisited, Physica A 232, 207 (1996).

[25] N. N. Bogoliubov, Lectures on Quantum Statistics (Gordon and Breach, New York, 1970)

[26] L. Lauck, A. R. Vasconcellos, and R. Luzzi, A Nonlinear Quantum Transport Theory, Physica A168, 789 (1990).

[27] J. G. Ramos, A. R. Vasconcellos, and R. Luzzi, On the Truncation Procedure in the Nonequilibrium Statistical Operator Method, IFGW-Unicamp Internal Reports (1997), and future publication.

[28] J. R. Madureira, A. R. Vasconcellos, R. Luzzi, J. Casas-Vázquez, and D. Jou, Evolution of Dissipative Processes in a Statistical Thermodynamic Approach I and II, IFGW-Unicamp Internal Report (1997), and J. Chem. Phys. 108, 7568 (1998); ibid. 108, 7580 (1998).

[29] J. R. Madureira, A. R. Vasconcellos, R. Luzzi, and L. Lauck, Markovian Kinetic Equations in a Nonequilibrium Statistical Ensemble Formalism, Phys. Rev. E, 57, 3637 (1998).

[30] J. R. Madureira, A. R. Vasconcellos, and R. Luzzi, A Nonequilibrium Statistical Grand-Canonical Ensemble: Description in terms of Flux Operators, IFGWUnicamp Internal Report (1997), and J. Chem. Phys., in press (1998). 
[31] S. V. Peletminskii and A. I. Sokolovskii, Flux Operators of Physical Variables and the Method of Quasiaverages, Math. Theor. Phys. (USSR) 18, 121 (1974).

[32] D. N. Zubarev and V. P. Kalashnikov, Extremal Properties of the Nonequilibrium Statistical Operator, Math. Theor. Phys. (USSR) 1, 108 (1970).

[33] R. Courant and D. Hilbert, Methods of Mathematical Physics (Wiley-Interscience, New York, 1953): Vol. I, pp. $184-186$.

[34] A. R. Vasconcellos, R. Luzzi and L. S. Garcia-Colin, A Microscopic Approach to Irreversible Thermodynamics I: General Theory, Phys. Rev. A 43, 6622 (1991); ibid. II: An Example from Semiconductors Physics, 43, 6633 (1991).

[35] A. R. Vasconcellos, A. C. Algarte, and R. Luzzi, Diffusion of Photoinjected Carriers in Plasma Nonequilibrium Semiconductors, Phys. Rev. B 48, 10873 (1993).

[36] A. R. Vasconcellos, R. Luzzi, and A. S. Esperidião, Damped Plasma Wave in Photoexcited Plasma in Semiconductors, Phys. Rev. B 52, 5021 (1995).

[37] A. R. Vasconcellos, R. Luzzi, D. Jou, and J. CasasVázquez, Second Sound Wave in Photoinjected Plasma in Semiconductors, Phys. Rev. B 52, 5030 (1995).

[38] D. Jou, J. Casas-Vázquez, and G. Lebon, Extended Irreversible Thermodynamics (Springer, Berlin, 1993; second edition 1996).

[39] P. M. Morse and H. Feshbach, Methods of Theoretical Physics (McGraw-Hill, New York, 1953).
[40] R. Luzzi, M. A. Scarparo, J. G. Ramos, A. R. Vasconcellos, M. L. Barros, Z. Zhiyao, and A. Kiel, Nonequilibrium Thermodynamics of Laser Thermal Stereolithography, J. Non-Equilib. Thermodyn. 22, 197 (1997).

[41] J. G. Ramos, A. R. Vasconcellos, and L. S. GarciaColin, A Thermo-Hydrodynamic Theory Based on Informational Statistical Thermodynamics, Braz. J. Phys. 27, 585 (1997).

[42] J. G. Ramos, A. R. Vasconcellos, and R. Luzzi, A Nonclassical Navier-Stokes Equation in the Framework of a Nonequilibrium Ensemble Formalism, IFGW-Unicamp Internal Report (1997), and future publication.

[43] R. Luzzi, A. R. Vasconcellos, J. Casas-Vázquez, and D. Jou, Characterization and Measurement of a Nonequilibrium Temperature-like Variable, Physica A 234, 699 (1997).

[44] R. Luzzi, A. R. Vasconcellos, D. Jou, and J. CasasVázquez, Thermodynamic variables in the Context of a Nonequilibrium Statistical Ensemble Approach, J. Chem. Phys. 107, 7383 (1997).

[45] J. R. Madureira, A. R. Vasconcellos, and R. Luzzi, On a Generalized Gibbs-Boltzmann Ensemble Formalism for Dissipative Systems, Proc. V Latin American Workshop on Nonlinear Phenomena, Physica A, in press (1998). 